\title{
Colour in the cracks
}

Our
microcapsule-
based scheme
provides an
autonomous
in situ
indication
of damage

Cracks in polymer coatings and composites can lead to faster degradation of the underlying substrates or a reduction in the mechanical performance of the composite over time. In the case of polymer-coated steel structures - for example, ships and bridges - the detection of cracks at an early stage is essential to prevent corrosion, particularly in marine settings. Now, Nancy Sottos and colleagues, at the University of Illinois at Urbana-Champaign, show the visual detection of mechanical damage in epoxy polymer coatings using microcapsules containing a chemical indicator. "Our microcapsule-based scheme provides an autonomous in situ indication of damage as small as $10 \mu \mathrm{m}$," says Sottos.

The microcapsules - themselves composed of polymer - are homogeneously embedded within amine-cured epoxy polymer coatings. When the polymer coatings are scratched, the microcapsules in the damaged region rupture and the chemical indicator is released into the epoxy coating. On reaction with residue amine groups in the polymer, the chemical indicator changes dramatically in colour, from light yellow to bright red. The indicator also precipitates out of solution on release from the microcapsule and, as a result, the colour change is localized in the damaged region. As expected, the intensity of the colour is greater if the scratch is deeper, as more microcapsules are ruptured and a greater amount of indicator is released.

"Key advances over previous systems include high contrast between the 'on' and 'off' states and the excellent long-term stability both before and after the damage event, with no false positives or bleaching," says Sottos. Damaged samples show the same colour intensity after 8 months, and fresh scratches made at this time in undamaged regions are of the same intensity as when the initial scratches were made 8 months previously. In addition, compared with other damage detection systems using chemical indicators, this method does not require additives, such as catalysts or colour developers, or activation with ultraviolet light, which may have an adverse effect on the properties of the original coating.

Sottos and colleagues show that their microcapsule system can be adapted for use in polymers without unreacted amine groups in the matrix: for example, in polydimethylsiloxane. This requires a dual-microcapsule approach with both a chemical indicator and amine-loaded microcapsules embedded in the polymer coating. Moreover, microcapsules containing an epoxy monomer can be added and, hence, the damage revealed by the chemical indicator can be subsequently healed.

"We are currently working on combining the detection of virgin damage with self-healing functionality and then using a secondary indicator to reveal that crack healing has occurred," says Sottos. The researchers are also developing different chemical indicators that could detect damage in various polymeric coatings. "We hope that this technology can be applied to commercial coatings in 3 to 5 years," concludes Sottos.

Alison Stoddart

ORIGINAL ARTICLE Li, W. et al. Autonomous indication of mechanical damage in polymeric coatings. Adv. Mater. http://dx.doi.org/10.1002/ adma.201505214 (2016)

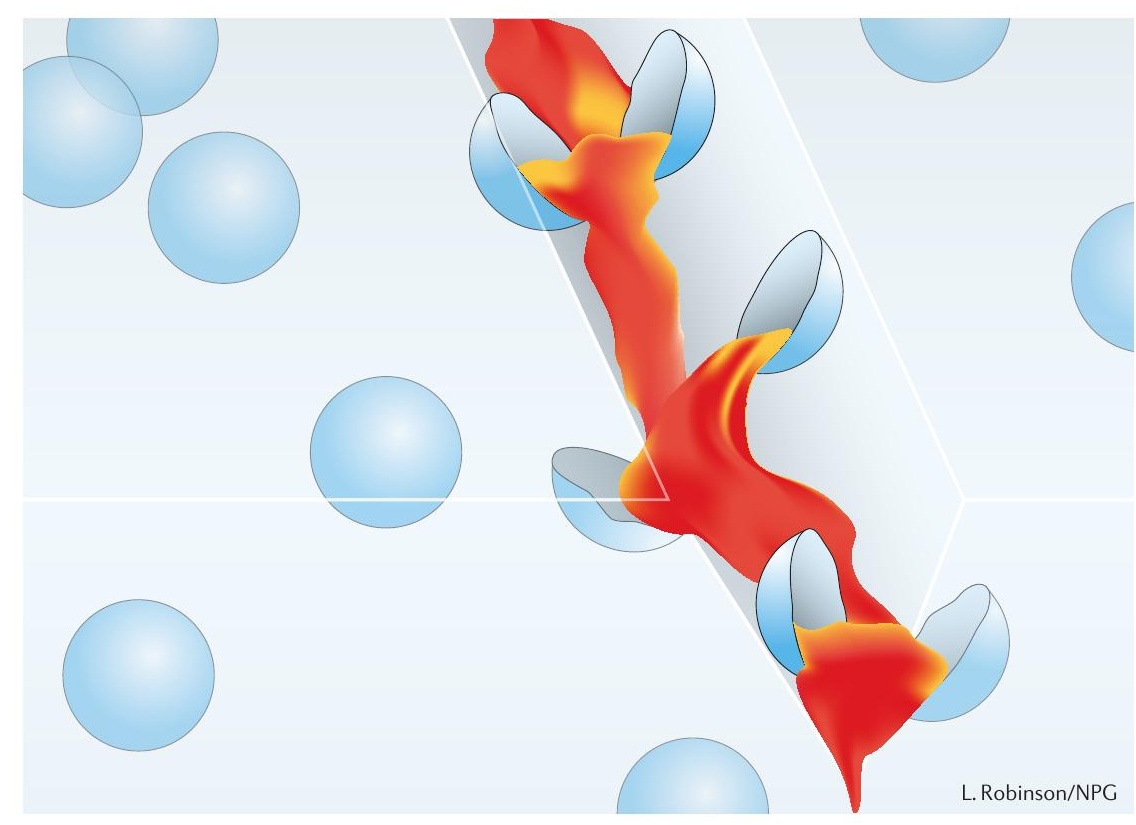

